\title{
Sunitinib for Metastatic Renal Cell Carcinoma: A Systematic Review and Meta-Analysis of Real-World and Clinical Trials Data
}

\author{
Michael Moran ${ }^{1} \cdot$ Dana Nickens $^{2} \cdot$ Katherine Adcock $^{3} \cdot$ Meg Bennetts $^{4} \cdot$ Arial Desscan $^{3} \cdot$ Natalie Charnley $^{5} \cdot$ Kate Fife $^{6}$
}

Published online: 12 July 2019

(c) The Author(s) 2019

\begin{abstract}
Background Randomized controlled trials (RCTs) have stringent inclusion criteria and may not fully represent patients seen in everyday clinical practice. Real-world data (RWD) can provide supportive evidence for the effectiveness of medical interventions in more heterogeneous populations than RCTs. Sunitinib is a widely used first-line treatment for patients with metastatic renal cell carcinoma (mRCC).

Objective This is the first comprehensive meta-analysis to evaluate the efficacy of sunitinib using the novel approach of combining RCTs and RWD.

Methods RCTs and RWD studies published between 2000 and 2017 were identified from PubMed, Ovid, MEDLINE, and EMBASE. Eligible studies contained a cohort of $\geq 50$ adult patients with $\mathrm{mRCC}$ receiving first-line sunitinib treatment. The meta-analysis combined RWD and RCT treatment groups, adjusting for data type (RCT or RWD). Recorded outcomes were median progression-free survival (mPFS), median overall survival (mOS), and objective response rate (ORR). Publication bias was assessed via review of funnel plots for each outcome measure. A random effects model to account for study heterogeneity was applied to each endpoint. Sensitivity analyses evaluated the robustness of the overall estimates.

Results Of the 3611 studies identified through medical database searches, 22 (15 RWD studies, 7 RCTs) met eligibility criteria and were analyzed. mPFS (18 studies), mOS (19 studies), and ORR (15 studies) were reported for aggregate measures based on 4815, 5321, and 4183 patients, respectively. Reported mPFS (RWD, 7.5-11.0 months; RCTs, 5.6-15.1 months) and ORR data (RWD, 14.0-34.6\%; RCTs, 18.8-46.9\%) were consistent with the overall confidence estimates (95\% confidence interval $[\mathrm{CI}])$ of $9.3(8.6-10.2)$ months and $27.9 \%$ (24.2-32.0), respectively. Reported mOS showed greater variation in RWD (6.8-33.2 months) compared with RCTs (21.8-31.5 months), with an overall confidence estimate (95\% CI) of 23.0 (19.2-27.6) months. Inspection of funnel plots and sensitivity analyses indicated that there was no publication bias for any efficacy endpoint. Sensitivity analyses showed no evidence of lack of robustness for mPFS, mOS, or ORR. Interpretation of these results is limited by differences in trial design, cohort characteristics, and missing data.

Conclusions This novel, comprehensive meta-analysis validates sunitinib as an effective first-line treatment for patients with $\mathrm{mRCC}$ in both RCTs and everyday clinical practice. The methodology provides a framework for future analyses combining data from RCTs and RWD.
\end{abstract}

Electronic supplementary material The online version of this article (https://doi.org/10.1007/s11523-019-00653-5) contains supplementary material, which is available to authorized users.

Michael Moran

michael.m.moran@pfizer.com

Pfizer GmbH, Linkstraße 10, 10785 Berlin, Germany

Pfizer Global, La Jolla, CA, USA

Pfizer Inc., London, UK

Pfizer R\&D UK Ltd, Sandwich, UK

5 Lancashire Teaching Hospitals, Lancashire, UK

6 Cambridge University Hospital, Cambridge, UK

\section{Key Points}

To assess if a drug works, clinical trials only include patients that meet certain criteria and therefore may not fully represent those patients seen in everyday clinical practice.

This study combined results from clinical trials and everyday clinical practice to provide a realistic estimate of how effective sunitinib is in treating an advanced type of kidney cancer, metastatic renal cell carcinoma.

Data from clinical trials and real-world clinical practice were similar, confirming that sunitinib is an effective first treatment for patients with metastatic renal cell carcinoma. 


\section{Introduction}

Sunitinib malate, an oral, multi-targeted receptor tyrosine kinase inhibitor (TKI), has been the gold-standard firstline treatment for metastatic renal cell carcinoma (mRCC) for the past 12 years. Sunitinib exerts an anti-angiogenic effect through inhibition of vascular endothelial growth factor (VEGF) receptors and platelet-derived growth factor (PDGF) receptors [1]. Most (approximately 80\%) RCC cases are of the clear cell histology subtype and are commonly associated with inactivation of the von Hippel-Lindau (VHL) tumor suppressor gene [2]. Inactivation of $V H L$ leads to the downstream overexpression of VEGFs and PDGFs, which promote tumor growth through increased angiogenesis. Targeting these pathways therefore represents a potentially effective strategy.

There are approximately 400,000 newly diagnosed cases of kidney cancer each year, with RCC accounting for the vast majority of cases [3]. Over $20 \%$ of patients with RCC are diagnosed with metastatic disease [4], which remains an important cause of cancer-related death with a 5-year survival rate of approximately $8 \%$ [5].

The approval of sunitinib in 2006 marked a major milestone in the treatment of $\mathrm{mRCC}$, ushering in the targeted therapy era. Previously, first-line mRCC treatment was with the cytokines interferon- $\alpha$ (IFN- $\alpha)$ or interleukin-2 (IL-2); however, these treatments had low response rates (approximately 10-14\%), limited survival, and poor tolerability [6, 7]. Following two phase II clinical trials in patients with cytokine-refractory mRCC, sunitinib received accelerated approval from the US Food and Drug Administration (FDA) [8-10]. In a phase III trial, sunitinib, compared with IFN- $\alpha$, was associated with significantly longer median progression-free survival (mPFS, 11 vs 5 months, respectively), objective response rate (ORR, $47 \%$ vs $12 \%$ ), and a clinically meaningful improvement in median overall survival (mOS, 26.4 vs 21.8 months). This established a new clinical standard of care for firstline mRCC treatment [11]. New medical interventions for mRCC, especially targeted therapies, are therefore often compared with sunitinib for efficacy and safety.

Available treatment options for $\mathrm{mRCC}$ have dramatically expanded over the past 12 years with the regulatory approvals of sorafenib, pazopanib, axitinib, cabozantinib, tivozanib (EU only), nivolumab, ipilimumab, lenvatinib, everolimus, temsirolimus, and bevacizumab. The promising outcomes reported with recently approved combination therapies nivolumab plus ipilimumab (EU and USA approved for intermediate and poor risk patients), avelumab plus axitinib (USA), and pembrolizumab plus axitinib (USA) are set to expand the treatment landscape for $\mathrm{mRCC}$ further [12-15]. However, sunitinib remains one of several recommended first-line treatments for patients with favorable risk mRCC [16-18]. Choosing the optimal sequence of treatment has subsequently become increasingly complex for the clinician and the patient $[19,20]$.

Randomized controlled trials (RCTs) are the basis of approval for medical interventions, but may not fully reflect populations seen in clinical practice [21-24]. Patients in oncology RCTs are generally younger, healthier, and predominantly of White ethnicity. Differences between RCT and realworld patient populations do not necessarily rule out equivalent efficacy, but may introduce doubt. If the result of an RCT is to be clinically useful, there must be a reasonable likelihood that the result can be replicated in a real-world clinical setting [25]. The applicability and generalizability (external validity) of RCTs can also be affected by many aspects of trial design, including but not limited to geographic location, patient selection, trial protocol versus routine practice, outcome measures, patient follow-up, and the management and reporting of adverse events (AEs) [25, 26]. Real-world data (RWD) may help dispel uncertainty in the generalizability of RCTs by providing supportive evidence, especially for patients who would not have been eligible for RCTs [27]. The work presented in this paper adds to the evidence supporting the use of RWD in clinical decision-making.

The objective of this analysis was to evaluate efficacy outcomes in both the RCT and real-world setting, providing clinicians with an overall estimate of sunitinib efficacy that may aid in treatment decision-making. To our knowledge, this is the first comprehensive meta-analysis to evaluate the efficacy of sunitinib using the novel approach of combining RCTs and RWD.

\section{Methods}

\subsection{Search Strategy and Selection Criteria}

This was a subgroup analysis of a systematic review and meta-analysis of TKI efficacy in RCTs and RWD studies. The Population, Interventions, Comparator, Outcomes, and Study Designs (PICOS) framework and Preferred Reporting Items for Systematic Reviews and Meta-Analyses (PRISMA) guidelines were used to inform study design and reporting [28]. RCTs and RWD studies of sunitinib as first-line treatment in patients with mRCC published between 2000 and 2017 were identified from a search of PubMed, Ovid, MEDLINE, and EMBASE. The literature search was completed in July 2018 and included only English language publications. Exclusion of non-English language publications is unlikely to impact systemic bias [29]. Studies eligible for this subgroup analysis contained a cohort of $\geq 50$ adult patients per treatment group. If more than one publication was found for the same study, 
only the most recent analysis was included. The database search strategy included the terms 'kidney,' 'renal,' 'carcinoma*,' 'cancer*, 'neoplasm*,' 'tumo?r*, 'malignan*, 'oncolog*,' 'metastatic*,' and 'metastasis*,' in combination with the terms 'first line,' ' 1 st line,' 'initial*,' 'treatment*,' 'therap*,' and 'intervention*'.

\subsection{Data Extraction and Analysis}

The literature search was conducted by two investigators (MM and KA). One investigator (KA) identified relevant data. Disagreements were reviewed via discussion until a consensus was reached. Data were extracted for analysis by a single investigator (DN). An independent check of the data against the source material was conducted for quality assurance (MB).

The meta-analysis combined RWD and RCT treatment groups, adjusting for data type (RCT or RWD). Recorded outcomes were mPFS, mOS, and ORR. A between-study random effect model, $\left(\sim N\left(0, \tau^{2}\right)\right)$, was applied to each endpoint to account for study heterogeneity $\left(\tau^{2}\right)$. Confidence limits for the estimates (95\% confidence interval [CI]) provided bounds within which the true combined RCT or RWD mean should lie for each efficacy outcome with $95 \%$ probability. Confidence limits for the prediction estimates (95\% CI) provided the range of possible values that should contain the RCT or RWD mean for a future sunitinib treatment group efficacy outcome with $95 \%$ probability.

\subsection{Bias and Sensitivity Analyses}

Publication bias for each efficacy endpoint was assessed via investigator review of funnel plots. Pre-specified sensitivity analyses were used to evaluate the robustness of the overall estimates and were conducted for each efficacy endpoint. To assess study heterogeneity, individual RWD studies were omitted one at a time and compared with RWD studies-only estimate, RCT-only estimate, and the final model estimate. The robustness of the random effects model was assessed by step-wise inflation of RWD variance and comparison with RWD studies-only estimate, RCT-only estimate, and the final model estimate.

\section{Results}

\subsection{Systematic Review}

A total of 3611 studies were identified through our medical database searches. Following screening to remove irrelevant studies, 68 potentially eligible studies were thoroughly evaluated. Of these, 35 studies met eligibility criteria for the analysis of first-line TKI efficacy in mRCC. Twenty-two studies (15 RWD, 7 RCTs) included at least one sunitinib treatment group and were included in the subgroup analysis (Fig. 1, Supplementary materials Table 1 and 2, see electronic supplementary material [ESM]). mPFS (18 studies), mOS (19 studies), and ORR (15 studies) were reported for aggregate measures based on 4815, 5321, and 4183 patients, respectively (Table 1). Of the investigated efficacy measures, ORR was the measure most commonly not reported.

All RCTs included in the analysis were multicenter studies that assessed tumor progression using Response Evaluation Criteria In Solid Tumors (RECIST) [11, 30-35]. All RCTs, with the exception of RECORD-3, included only patients with clear cell histology. Comparator interventions included cabozantinib [31], everolimus [30, 32], pazopanib [33], IFN- $\alpha$ [11], and a combination of sunitinib with IMA901, a multi-peptide cancer vaccine [35]. One RCT compared an alternative sunitinib dosing schedule with the 4 weeks on and 2 weeks off (4/2) dosing schedule [34]. Both arms of this trial were included in the analysis.

RWD studies used a variety of sources to extract data, including national and local cancer registries, insurance databases, pharmacy databases, and patient medical records [36-50]. Studies were from a wide range of countries, including USA, Canada, Australia, Spain, France, Greece, Italy, Netherlands, Czech Republic, China, Japan, Korea, and India. The majority of RWD studies were retrospective analyses, with only two studies of prospective design [41, 49].

\subsection{Meta-Analysis}

\subsubsection{Median Progression-Free Survival}

Reported mPFS values ranged from 7.5 to 11.0 months in RWD studies (11 studies) and from 5.6 to 15.1 months in RCTs (7 studies). In RCTs, reported mPFS showed little variation with the exception of 15.1 and 5.6 months reported by Rini et al. [35] and Choueiri et al. [31], respectively (Fig. 2). In RWD studies, reported mPFS was largely consistent and fell within a small range of 3.5 months. The combined RWD and RCT data confidence estimate was 9.3 months (95\% CI 8.6-10.2) and the combined prediction estimate was 9.3 months (95\% CI 7.0-12.5) (Fig. 2, Table 2).

\subsubsection{Median Overall Survival}

Reported mOS showed greater variation in RWD (6.8-33.2 months, 14 studies) compared with RCTs (21.8-31.5 months, 5 studies; Fig. 3). The majority of mOS values reported in RCTs were similar to the combined 
Fig. 1 PRISMA flow diagram of the literature search process. PRISMA Preferred Reporting Items for Systematic Reviews and Meta-Analyses, $R C T$ randomized controlled trial, $R W D$ real-world data, $T K I$ tyrosine kinase inhibitor

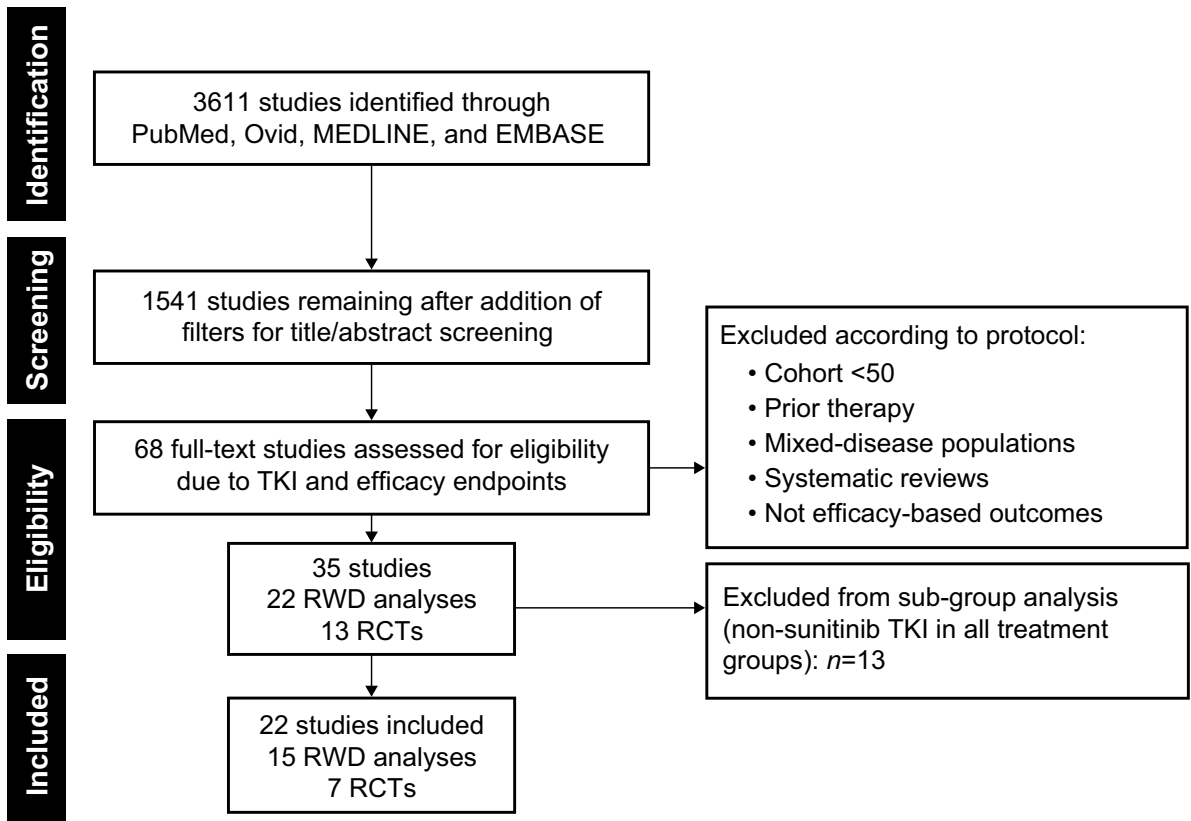

Table 1 Number of studies, treatment arms, and patients included in analyses

\begin{tabular}{|c|c|c|c|c|c|c|}
\hline & \multicolumn{2}{|c|}{ Studies, $n$} & \multicolumn{2}{|c|}{ Treatment groups, $n$} & \multicolumn{2}{|c|}{ Patients, $n$} \\
\hline & RWD & RCT & RWD & RCT & RWD & RCT \\
\hline $\mathrm{mPFS}$ & 11 & 7 & 11 & 8 & 3098 & 1717 \\
\hline $\mathrm{mOS}$ & 14 & 5 & 14 & 6 & 3972 & 1349 \\
\hline ORR & 10 & 5 & 10 & 6 & 2694 & 1489 \\
\hline
\end{tabular}

$m O S$ median overall survival, $m P F S$ median progression-free survival, $O R R$ objective response rate, $R C T$ randomized controlled trial, $R W D$ realworld data

confidence estimate of 23.0 months (95\% CI 19.2-27.6). A similar pattern was observed for the majority of RWD studies reporting mOS (Fig. 3). One RWD study by De Groot et al. reported a particularly poor mOS value of 6.8 months, which was substantially shorter than other reported values. In a study by Rini et al., mOS was not reached with sunitinib treatment during a median follow-up of 33.3 months and was not included in the combined estimates [35]. The combined prediction estimate for mOS was 23.0 months (95\% CI 11.2-47.4; Table 2).

\subsubsection{Objective Response Rate}

The combined confidence estimate for ORR was 27.9\% (95\% CI 24.2-32.0). Overall, reported ORR data were mostly consistent with the combined confidence estimate (Fig. 4, Table 2) for both RCTs (18.8-46.9\%, 5 studies) and RWD (14.0-34.6\%, 10 studies). Motzer et al. reported a particularly high ORR of $46.9 \%$ from an RCT sunitinib treatment arm [11]. The combined prediction estimate for ORR was $27.9 \%$ (95\% CI 16.2-43.6; Table 2).

\subsubsection{Sensitivity Analyses}

Pre-specified sensitivity analyses showed no evidence of lack of robustness in the combined final confidence estimate for mPFS, mOS, or ORR (Supplementary Figs. 1-3, see ESM). Investigation of funnel plots revealed no evidence of publication bias.

\section{Discussion}

This comprehensive systematic review and meta-analysis used the novel approach of combining RWD and RCT data to examine the effectiveness of sunitinib in both RCTs and everyday clinical practice. The stringent eligibility criteria and intensive monitoring applied in RCTs allows for the generation of robust evidence to answer a specific clinical question in a clearly defined population. An often unavoidable result is that little or no evidence is generated to support clinical decision-making for those groups of patients who did not meet eligibility criteria. In 


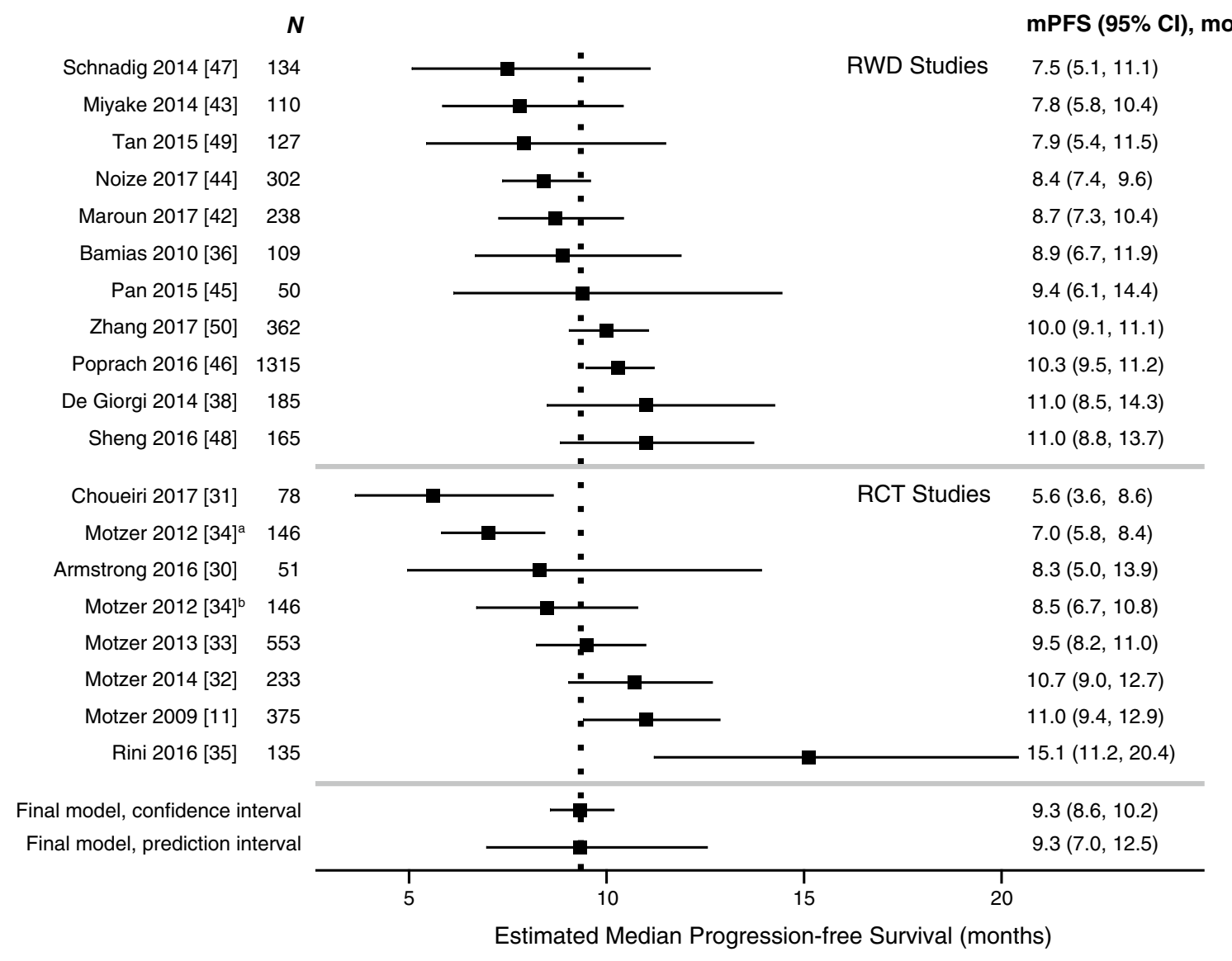

Fig. 2 Forest plot of mPFS final model confidence and prediction intervals and reported individual study arm mPFS. $C I$ confidence interval, $m P F S$ median progression-free survival, $R C T$ randomized

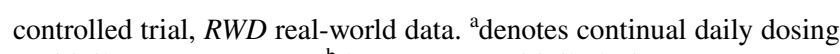
sunitinib treatment group, ${ }^{b}$ denotes $4 / 2$ sunitinib dosing

Table 2 Final model results of sunitinib efficacy endpoints for RWD, RCTs, and combined data

\begin{tabular}{|c|c|c|c|c|}
\hline & $\begin{array}{l}\text { RWD-only confidence } \\
\text { estimate }^{\text {a }}(95 \% \mathrm{CI})\end{array}$ & $\begin{array}{l}\text { RCT-only confidence } \\
\text { estimate }^{\mathrm{a}}(95 \% \text { CI) }\end{array}$ & $\begin{array}{l}\text { Combined confidence } \\
\text { estimate }^{\mathrm{a}}(95 \% \mathrm{CI})\end{array}$ & $\begin{array}{l}\text { Combined prediction } \\
\text { estimate }^{\mathrm{a}}(95 \% \mathrm{CI})\end{array}$ \\
\hline mPFS & $9.4(8.7-10.2)$ & $9.3(7.7-11.2)$ & $9.3(8.6-10.2)$ & $9.3(7.0-12.5)$ \\
\hline mOS & $20.8(16.5-26.0)$ & $25.7(22.9-28.7)$ & $23.0(19.2-27.6)$ & $23.0(11.2-47.4)$ \\
\hline ORR & $26.1 \%(22.3-30.3)$ & $29.7 \%(22.9-37.6)$ & $27.9 \%(24.2-32.0)$ & $27.9 \%(16.2-43.6)$ \\
\hline
\end{tabular}

Confidence and prediction estimates are based on a random effects model

${ }^{a}$ Months, unless otherwise stated

$C I$ confidence interval, $m O S$ median overall survival, $m P F S$ median progression-free survival, $O R R$ objective response rate, $R C T$ randomized controlled trial, $R W D$ real-world data

contrast, RWD can provide supportive evidence for treatment strategies or patient groups not assessed in RCTs. This may be particularly important for mRCC, where the recent expansion of available treatments has made clinical decision-making more complex. Our results confirm sunitinib to be an effective first-line treatment in patients with $\mathrm{mRCC}$ in both the RCT and real-world settings. In a realworld or RCT setting, our analysis predicts that sunitinib treatment in patients with $\mathrm{mRCC}$ will result in an average of 9.3 months PFS, 23.0 months OS, and a $27.9 \%$ ORR.

Sunitinib is often used as the comparator in RCTs for new first-line mRCC treatments. In the phase III COMPARZ trial, pazopanib achieved non-inferiority compared with sunitinib, with an mPFS of 8.4 months for pazopanib and 9.5 months for sunitinib [33]. The authors concluded that pazopanib was favored over sunitinib in terms of safety 


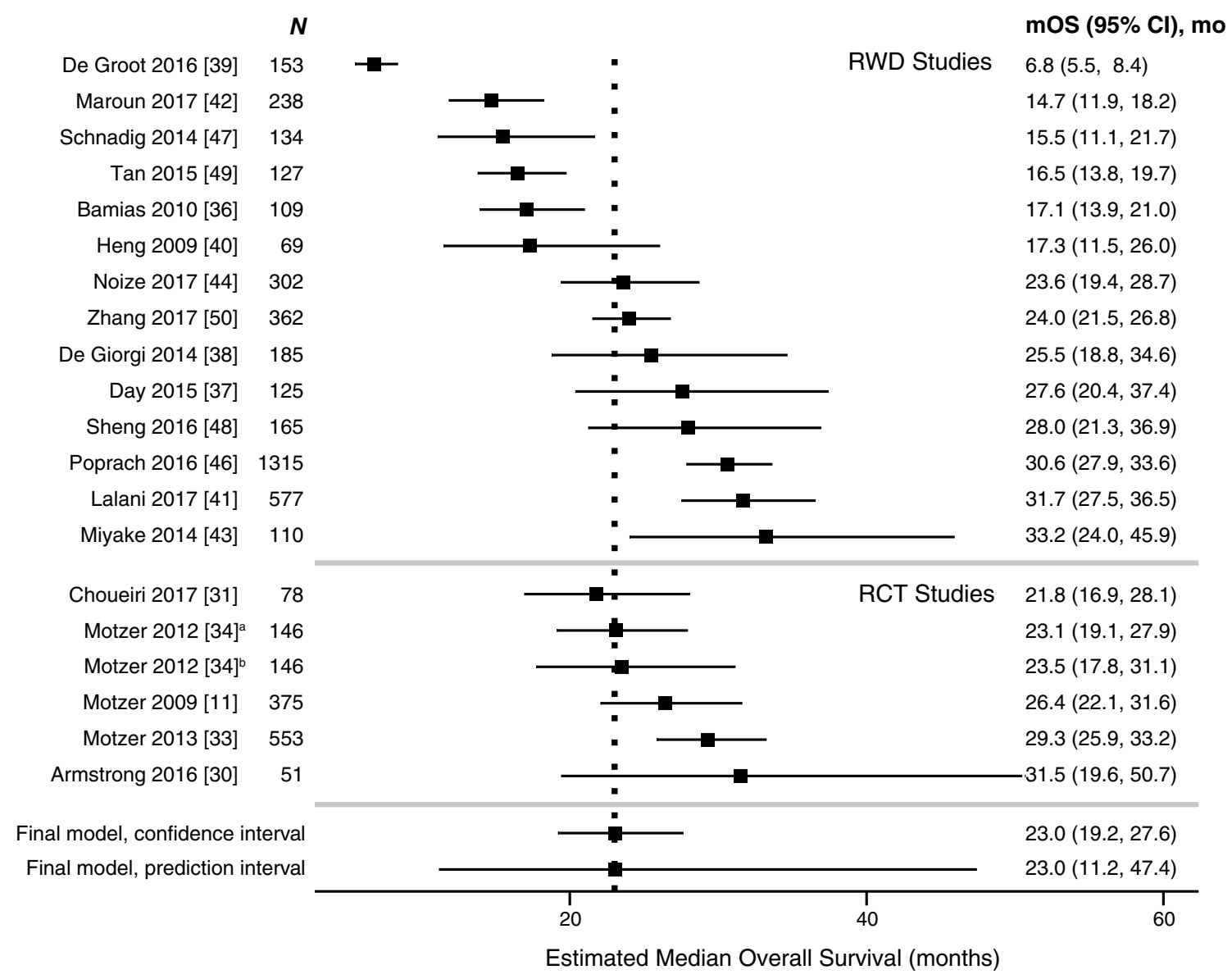

Fig. 3 Forest plot of mOS final model confidence and prediction intervals and reported individual study arm mOS. CI confidence interval, $m O S$ median overall survival, $R C T$ randomized controlled

and patient quality of life (QoL) [33]. Although the mPFS reported in the COMPARZ trial is in line with our results, bias inherent in the trial design means that the results from the trial must be interpreted with caution. Specifically, efficacy and patient satisfaction measures were collected at different times. Sunitinib was administered according to the once-daily $4 / 2$ dosing schedule. Efficacy evaluations were collected on day 42 , the final day of week 6 of a sunitinib dosing cycle, when efficacy is at its poorest (following 2 weeks off sunitinib). Patient QoL surveys, on the other hand, were undertaken on day 28 , the final day of week 4 of a sunitinib treatment cycle, when AEs are most likely to be at their most significant [33]. Outcomes may therefore have been skewed in favor of pazopanib.

The RWD studies of sunitinib versus pazopanib have differed in their conclusions. A large independent study found no difference in PFS, OS, or ORR between sunitinib and pazopanib [51]. These values were generally consistent with the phase III RCT. Another independent study found that in Canadian patients, sunitinib was associated with an trial, $R W D$ real-world data. ${ }^{a}$ denotes $4 / 2$ sunitinib dosing, ${ }^{b}$ denotes continual daily dosing sunitinib treatment group

improved OS of 31.7 months compared with pazopanib OS of 20.6 months [41]. In contrast, an analysis of US Medicare data found that treatment with pazopanib versus sunitinib was a positive prognostic factor [52]. Such divergent results reinforce the value of RCTs in controlling for known and unknown confounders. Furthermore, disagreement between RCTs and RWD can make it difficult for clinicians to unravel what the true effect of treatment may be in a specific population. Clinicians must not only be experts in their field of medicine, but also in interpreting the integrity of RWD or the design and reliability of an RCT [53]. A strength of our study is that both RWD and data from RCTs are included in the final model, providing a robust estimate of expected sunitinib efficacy.

More recently, the development of immune checkpoint inhibitors and their use in combination therapy will further change the landscape of mRCC first-line treatment. A phase III study of nivolumab plus ipilimumab in patients with intermediate- or poor-risk status reported mPFS of 11.6 months and a 12 -month OS rate of $80 \%$ [13]. As 


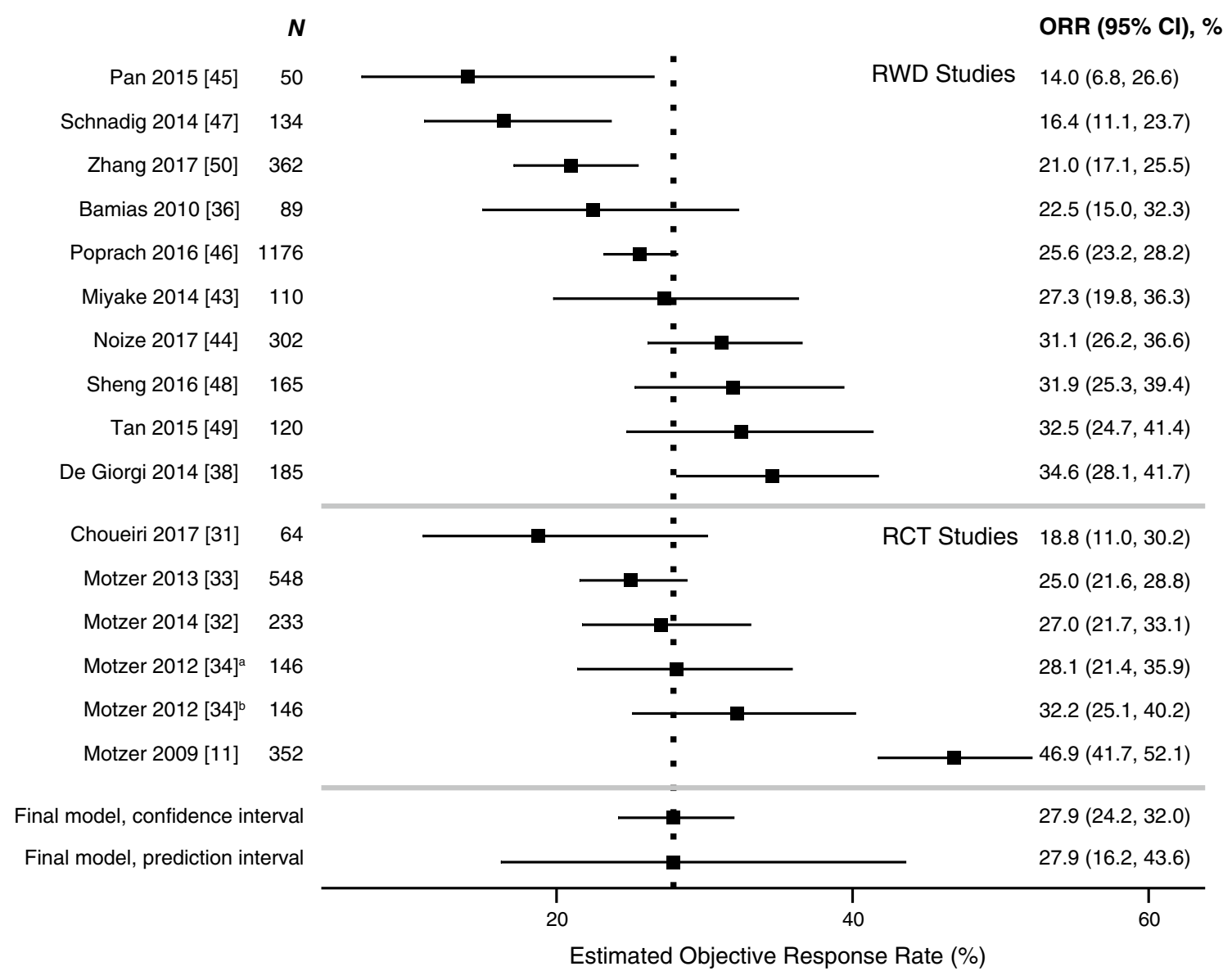

Fig. 4 Forest plot of ORR final model confidence and prediction intervals and reported individual study arm ORR. CI confidence interval, $O R R$ objective response rate, $R C T$ randomized controlled

comparator, sunitinib was reported to achieve an mPFS of 8.4 months and a 12 -month OS rate of $72 \%$. Exploratory analyses in 249 patients from the same RCT found that in patients with favorable risk status, sunitinib was associated with significantly higher ORR (52\%) and longer mPFS (25.1 months) compared with nivolumab plus ipilimumab (29\% and 15.3 months, respectively). Treatment-related AEs (of any grade) were very common in patients receiving sunitinib (97\%) or nivolumab plus ipilimumab (93\%). Of the patients who received nivolumab plus ipilimumab, $80 \%$ had treatment-related, immune-mediated AEs, with $35 \%$ requiring high-dose glucocorticoids. To optimize therapy with immune checkpoint inhibitors, management of treatment-related, immune-mediated AEs will need to be effectively balanced with efficacy. Over 10 years of clinical experience with sunitinib has led to the development of alternative strategies to manage treatment-related AEs (discussed below). Clinical experience with immune checkpoint inhibitors in $\mathrm{mRCC}$ is rapidly increasing and may similarly lead to new insights into the management of treatment-related, immune-mediated AEs. trial, $R W D$ real-world data. adenotes continual daily dosing sunitinib treatment group, ${ }^{b}$ denotes $4 / 2$ sunitinib dosing

The results of two other phase III studies have recently been reported for the combinations avelumab plus axitinib and pembrolizumab plus axitinib [14, 15]. In each study, mPFS, ORR, and OS estimates were superior with the immune checkpoint inhibitor plus TKI combination compared with sunitinib. The reported mPFS (8.4 and 11.1 months) and ORR (25.7\% and $35.7 \%$ ) for sunitinib in these studies were consistent with the confidence and prediction estimates from our analysis $[14,15]$. The role of sunitinib in future first- and subsequent-line mRCC therapy remains to be clearly defined. Although combination therapy with sunitinib and immune checkpoint inhibitors is being explored [54], sunitinib is mostly being used as the comparator in RCTs. The future of first-line sunitinib treatment may therefore be in patients unable to receive immunotherapy, or patients with a favorable risk status.

Sunitinib was approved for the treatment of advanced RCC by the FDA at a recommended dose of $50 \mathrm{mg}$ administered once daily on a $4 / 2$ dosing schedule. The $50 \mathrm{mg}$ oncedaily 4/2 dosing schedule was originally selected for future investigation based on phase I solid tumor studies in a small 
number of patients [55, 56]; however, in phase III RCTs, the 4/2 dosing schedule was associated with substantial AEs [11, 33]. Furthermore, RWD suggests that treatment discontinuation due to treatment-related AEs occurs sooner in clinical practice than in RCTs [47]. Alternative dosing schedules were investigated with the aim of achieving a better safety profile, allowing more patients to continue treatment for longer, and potentially improving outcomes. An RWD analysis that compared sunitinib 4/2 dosing with 2 weeks on treatment and 1 week off $(2 / 1)$ found that mPFS was significantly longer (9.5 vs 11.2 months), the incidence of specific AEs lower, and health-related QoL higher in patients receiving $2 / 1$ dosing [45]. However, the number of patients receiving $2 / 1$ dosing was low $(n=32)$. A recent analysis of nearly 300 patients from a German registry found that, when compared with $4 / 2$ dosing, sunitinib dose modification reduced the frequency of AEs and significantly increased mPFS (6.0 vs 15.1 months) and OS (13.7 vs 38.1 months) [57]. Notably, patients who received sunitinib dose modification were older, but had a more favorable risk status compared with patients receiving $4 / 2$ dosing, which may explain why these measures fall outside the confidence estimates reported herein. RCTs have also examined alternative dosing strategies including 2/1 dosing (phase II RESTORE study) [58] and continuous daily dosing (CDD) at a reduced dose of $37.5 \mathrm{mg} /$ day with no off-treatment phase (phase II RENAL effect study) [34]. Overall, 2/1 dosing appears to be associated with reduced toxicity and potential improvement in outcomes compared with 4/2 dosing, whereas CDD appears to provide no efficacy or safety advantage.

Despite the ability of targeted therapy to improve survival in patients with $\mathrm{mRCC}$, uptake of targeted therapy in eligible patients appears to be sub-optimal, with approximately one-third of patients receiving no targeted therapy [39]. In particular, patients aged $\geq 65$ years are less likely to receive targeted therapy than younger patients [39]. Although elderly patients constitute a growing proportion of mRCC patients, they are one patient group often poorly represented in RCTs [23]. In treating elderly patients, clinicians must find a balance between improving patient survival and managing treatment-related AEs. This can prove complex as elderly patients are likely to have significant comorbidities and the evidence base for their treatment is limited. In the pivotal sunitinib phase III trial, only $13 \%$ of patients were aged $\geq 70$ years [11]. It would be reasonable to therefore question if the reported efficacy and safety of sunitinib was applicable to the elderly population. Our analysis included two RWD studies in elderly patients; each study population had a median age of 74 years and mPFS and mOS were consistent with the combined confidence estimate and phase III RCT $[11,38,46]$. Furthermore, several RWD studies have found no significant difference in PFS or OS between patients aged $\geq 70$ years and those aged $<70$ years
[46, 59-61]. Dose reductions or alternative dosing schedules are, however, more common in elderly patients compared with younger patients $[38,46]$.

Certain ethnic groups are also poorly represented in RCTs. Our analysis included several RWD studies in Asian populations that were of particular interest [43, 45, 48-50]. Studies conducted in Asian populations reported 7.8-11.0 months mPFS, 16.5-33.2 months mOS, and 21-33\% ORR. Overall, efficacy measures in these studies were generally comparable with those reported in RCTs as well as our combined confidence estimate; however, compared with Western populations, Japanese and Asian patients appear to be more sensitive to sunitinib, resulting in frequent dose reductions $[43,49]$. In these studies, dose reductions to mitigate AEs occurred in approximately $90 \%$ of patients. Decreasing the dose below 50-mg 4/2 dosing substantially reduced the incidence of AEs, including grade 3 and 4 toxicities. In a subgroup analysis of the COMPARZ trial, sunitinib was well tolerated in Asian patients; however, a slightly higher proportion of Asian patients required sunitinib dose modification compared with non-Asian patients [62]. The incidence and severity of AEs also differed between Asian and non-Asian patients, which may be due to differences in absorption and metabolism [62].

Patients with $\mathrm{mRCC}$ seen in everyday clinical practice are more likely to have poor-risk disease by Memorial Sloan Kettering Cancer Center (MSKCC) criteria and impaired performance status (Eastern Cooperative Oncology Group, ECOG) compared with patients enrolled in phase III trials [22]. Performance or risk status can have a dramatic effect on survival outcomes and may account for some of the variation in reported survival outcomes. Sunitinib efficacy outcomes reported by Schnadig et al. and Noize et al. were generally lower than those reported in RCTs and our combined confidence estimates; however, there were greater numbers of poor-risk patients and fewer favorable-risk patients in these studies compared with RCTs [44, 47]. An RWD study by Rini et al. that reported a particularly efficacious mPFS of 15.1 months with mOS not reached, may have been influenced by a large proportion of patients with favorableto-intermediate International Metastatic Renal Cell Carcinoma Database Consortium (IMDC) risk [35]. Choueiri et al. reported an mPFS with sunitinib of 5.6 months in a phase II trial in patients with intermediate or poor risk by IMDC criteria [31]. This was considerably lower than all other reported values for mPFS for both RWD and RCTs. Over a third of patients enrolled in the study by Choueiri et al. had bone metastases. Furthermore, patients with stable brain metastases were also eligible, which may partly account for the shorter reported mPFS. Consistent with these studies, the sunitinib global expanded-access trial, which provided access to sunitinib for $>4500$ patients ineligible for the registration-directed RCTs, found that MPFS and 
mOS varied according to IMDC and MSKCC criteria [63]. Patients in MSKCC poor, intermediate, and favorable groups had an mPFS of 5.4, 10.6, and 15.4 months and an mOS of 9.1, 20.0, and 56.5 months, respectively [63]. Given the pronounced effect on survival, the proportion of patients with poor, intermediate, or favorable risk status must be considered when interpreting the results of RCTs and RWD.

Patients with brain metastases, organ dysfunction, prior or concurrent malignancies, and non-clear cell histology are commonly ineligible for mRCC RCTs. As might be expected, clinical outcomes in patients who were ineligible for phase III trials have been reported to be inferior compared with those patients who met eligibility criteria [20, 64-66]. Reported mPFS of trial-eligible versus trial-ineligible patients with $\mathrm{mRCC}$ receiving targeted treatment ranged from 8.6 to 11.0 and from 5.2 to 6.5 months, respectively [20, 64, 65]. The difference in reported mOS appeared to be more substantial, with trial-eligible mOS ranging from 26.0 to 29.2 months compared with 8.5-14.6 months in trialineligible patients [20,64-66]. Exclusion of these patient groups from RCTs can make it difficult for clinicians to make informed decisions on the optimal course of treatment as well as manage patient outcome expectations. Our results primarily apply to patients with clear cell histology, with all but one RCT (RECORD-3) excluding patients with nonclear cell RCC. The majority of RWD studies predominantly consisted of patients with clear cell histology (approximately $>80 \%$ of patients), although a minority of patients with other histologies were also included. Non-clear cell mRCC is generally less responsive to VEGF-targeted therapy than clear cell mRCC; however, reported mPFS values for sunitinib range from 6.1 to 8.3 months [67], which overlap with the combined confidence estimates reported in our study. The sunitinib global expanded-access trial reported that patients with mRCC and brain metastases had an mPFS of 5.3 months [63]. Although this falls outside of our combined confidence estimate, it is still likely to represent a clinically meaningful response in this group of patients.

The main strengths of our study are the pre-specified systematic literature review and meta-analysis, which used the novel approach of combining RCT data and RWD to address the common problem of generalizability associated with RCTs. In addition, there was no evidence of publication bias and sensitivity analyses showed little variation compared with the overall estimates. Although accounted for in the meta-analysis random effects model, the high level of heterogeneity in study design is the main limitation of our study. Approaches to dosing strategy, the timing of study assessments, and variation in cohort characteristics differed considerably. Selection criteria specifically restricted patients to those who received first-line sunitinib treatment; however, it is possible that some RWD studies did not consider previous cytokine therapy as previous targeted therapy.
Patients previously treated with cytokines were not included in our analysis when this information was provided in the published papers. Missing data and associated imputations from RWD studies may have also been a source of error. For example, a RWD study by De Groot et al. reported an mOS of 6.8 months in patients treated with sunitinib, which was substantially lower than all other reported values. Missing data from this registry-based study meant that patients who were not eligible might have been included in the analysis [39]. As may be expected, patient demographics and baseline clinical features in RWD studies were of greater heterogeneity than in RCTs. These studies may more accurately represent those seen in everyday clinical practice than RCTs; however, there were still relatively few data on patients aged $\geq 70$ years. Although it was not the aim of this analysis to differentiate outcomes by co-morbidities, it should be noted that real-world efficacy data are very limited for first-line sunitinib treatment in patients with chronic kidney disease or other co-morbidities. Furthermore, data on measurable disease (by RECIST) and adequate organ function may be missing from some sources of RWD [39], making interpretation of mPFS and ORR difficult. Lastly, there was inconsistency between studies in the detail reported for the methods used to assess efficacy.

\section{Conclusions}

This novel, comprehensive meta-analysis validates the effectiveness of sunitinib as first-line treatment for patients with mRCC in both RCTs and everyday clinical practice. RWD were generally consistent with RCT data and may provide supportive evidence for patient groups often not included in RCTs, including elderly patients and under-represented ethnic groups. Survival outcomes can be expected to differ according to risk group and the presence of comorbidities such as brain metastases, but still represent a clinical benefit in these patients. Overall, first-line sunitinib treatment in patients with mRCC is estimated to result in 9.3 months mPFS, 23.0 months mOS, and 27.9\% ORR.

Acknowledgements Medical writing support was provided by Leon Adams, PhD, of Engage Scientific Solutions, and funded by Pfizer.

\section{Compliance with ethical standards}

Funding This study was sponsored by Pfizer Inc.

Disclosures Michael Moran, Dana Nickens, and Meg Bennetts are employees of Pfizer Inc. Arial Desscan is currently an employee of Pfizer (undergraduate program). Katherine Adcock was an employee of Pfizer between Sept 2017 and 2018 (undergraduate program). Natalie Charnley has received honoraria for lecture fees and advisory boards from Pfizer, Ipsen, and Janssen. Kate Fife has received honoraria for advice or lecture fees from BMS, Pfizer, Esai, Novartis, and Roche. 
Data Sharing Statement Upon request, and subject to certain criteria, conditions, and exceptions (see https://www.pfizer.com/scien ce/clinical-trials/trial-data-and-results for more information), Pfizer will provide access to individual de-identified participant data from Pfizer-sponsored global interventional clinical studies conducted for medicines, vaccines, and medical devices (1) for indications that have been approved in the US and/or EU or (2) in programs that have been terminated (i.e., development for all indications has been discontinued). Pfizer will also consider requests for the protocol, data dictionary, and statistical analysis plan. Data may be requested from Pfizer trials 24 months after study completion. The de-identified participant data will be made available to researchers whose proposals meet the research criteria and other conditions, and for which an exception does not apply, via a secure portal. To gain access, data requestors must enter into a data access agreement with Pfizer.

Open Access This article is distributed under the terms of the Creative Commons Attribution-NonCommercial 4.0 International License (http://creativecommons.org/licenses/by-nc/4.0/), which permits any noncommercial use, distribution, and reproduction in any medium, provided you give appropriate credit to the original author(s) and the source, provide a link to the Creative Commons license, and indicate if changes were made.

\section{References}

1. Mendel DB, Laird AD, Xin X, Louie SG, Christensen JG, Li $\mathrm{G}$, et al. In vivo antitumor activity of SU11248, a novel tyrosine kinase inhibitor targeting vascular endothelial growth factor and platelet-derived growth factor receptors: determination of a pharmacokinetic/pharmacodynamic relationship. Clin Cancer Res. 2003;9:327-37.

2. Maynard MA, Ohh M. Von Hippel-Lindau tumor suppressor protein and hypoxia-inducible factor in kidney cancer. Am J Nephrol. 2004;24:1-13. https://doi.org/10.1159/000075346.

3. Bray F, Ferlay J, Soerjomataram I, Siegel RL, Torre LA, Jemal A. Global cancer statistics 2018: GLOBOCAN estimates of incidence and mortality worldwide for 36 cancers in 185 countries. CA Cancer J Clin. 2018;68:394-424. https://doi.org/10.3322/ caac. 21492 .

4. Dabestani S, Thorstenson A, Lindblad P, Harmenberg U, Ljungberg B, Lundstam S. Renal cell carcinoma recurrences and metastases in primary non-metastatic patients: a population-based study. World J Urol. 2016;34:1081-6. https://doi. org/10.1007/s00345-016-1773-y.

5. The American Cancer Society. Survival rates for kidney cancer by stage. https://www.cancer.org/cancer/kidney-cancer/detec tion-diagnosis-staging/survival-rates.html Accessed Dec 2018.

6. Fyfe G, Fisher RI, Rosenberg SA, Sznol M, Parkinson DR, Louie AC. Results of treatment of 255 patients with metastatic renal cell carcinoma who received high-dose recombinant interleukin-2 therapy. J Clin Oncol. 1995;13:688-96. https://doi. org/10.1200/JCO.1995.13.3.688.

7. Minasian LM, Motzer RJ, Gluck L, Mazumdar M, Vlamis $\mathrm{V}$, Krown SE. Interferon alfa-2a in advanced renal cell carcinoma: treatment results and survival in 159 patients with longterm follow-up. J Clin Oncol. 1993;11:1368-75. https://doi. org/10.1200/JCO.1993.11.7.1368.

8. Motzer RJ, Michaelson MD, Redman BG, Hudes GR, Wilding G, Figlin RA, et al. Activity of SU11248, a multitargeted inhibitor of vascular endothelial growth factor receptor and platelet-derived growth factor receptor, in patients with metastatic renal cell carcinoma. J Clin Oncol. 2006;24:16-24. https://doi. org/10.1200/JCO.2005.02.2574.

9. Motzer RJ, Michaelson MD, Rosenberg J, Bukowski RM, Curti BD, George DJ, et al. Sunitinib efficacy against advanced renal cell carcinoma. J Urol. 2007;178:1883-7. https://doi. org/10.1016/j.juro.2007.07.030.

10. Motzer RJ, Rini BI, Bukowski RM, Curti BD, George DJ, Hudes GR, et al. Sunitinib in patients with metastatic renal cell carcinoma. JAMA. 2006;295:2516-24. https://doi.org/10.1001/ jama.295.21.2516.

11. Motzer RJ, Hutson TE, Tomczak P, Michaelson MD, Bukowski RM, Oudard S, et al. Overall survival and updated results for sunitinib compared with interferon alfa in patients with metastatic renal cell carcinoma. J Clin Oncol. 2009;27:3584-90. https://doi.org/10.1200/JCO.2008.20.1293.

12. Choueiri TK, Larkin J, Oya M, Thistlethwaite F, Martignoni M, Nathan P, et al. Preliminary results for avelumab plus axitinib as first-line therapy in patients with advanced clear-cell renal-cell carcinoma (JAVELIN Renal 100): an open-label, dose-finding and dose-expansion, phase 1b trial. Lancet Oncol. 2018;19:45160. https://doi.org/10.1016/S1470-2045(18)30107-4.

13. Motzer RJ, Tannir NM, McDermott DF, Aren Frontera O, Melichar B, Choueiri TK, et al. Nivolumab plus ipilimumab versus sunitinib in advanced renal-cell carcinoma. N Engl J Med. 2018;378:1277-90. https://doi.org/10.1056/NEJMoa1712126.

14. Motzer RJ, Penkov K, Haanen J, Rini B, Albiges L, Campbell MT, et al. Avelumab plus axitinib versus sunitinib for advanced renal-cell carcinoma. N Engl J Med. 2019;380(12):1103-15. https://doi.org/10.1056/NEJMoa1816047.

15. Rini BI, Plimack ER, Stus V, Gafanov R, Hawkins R, Nosov D, et al. Pembrolizumab plus axitinib versus sunitinib for advanced renal-cell carcinoma. N Engl J Med. 2019;380(12):1116-27. https://doi.org/10.1056/NEJMoa1816714.

16. Escudier B, Porta C, Schmidinger M, Rioux-Leclercq N, Bex A, Khoo V, et al. Renal cell carcinoma: ESMO clinical practice guidelines for diagnosis, treatment and follow-up. Ann Oncol. 2019;30(5):706-20. https://doi.org/10.1093/annonc/mdz056.

17. European Association of Urology. Renal Cell Carcinoma Guidelines. 2019. https://uroweb.org/guideline/renal-cell-carcinoma/. Accessed May 2019.

18. National Comprehensive Cancer Network. NCCN clinical practice guidelines in oncology: kidney cancer. Version 3. 2019. https://www.nccn.org/professionals/physician_gls/defau 1t.aspx\#site. Accessed May 2019.

19. Zarrabi K, Fang C, Wu S. New treatment options for metastatic renal cell carcinoma with prior anti-angiogenesis therapy. J Hematol Oncol. 2017;10:38. https://doi.org/10.1186/s1304 5-016-0374-y.

20. Goebell PJ, Staehler M, Muller L, Nusch A, Scheffler M, Sauer $\mathrm{A}$, et al. Changes in treatment reality and survival of patients with advanced clear cell renal cell carcinoma-analyses from the german clinical RCC-registry. Clin Genitourin Cancer. 2018. https ://doi.org/10.1016/j.clgc.2018.06.006.

21. Kaptchuk TJ. The double-blind, randomized, placebo-controlled trial: gold standard or golden calf? J Clin Epidemiol. 2001;54:541-9.

22. Mitchell AP, Harrison MR, Walker MS, George DJ, Abernethy AP, Hirsch BR. Clinical trial participants with metastatic renal cell carcinoma differ from patients treated in real-world practice. J Oncol Pract. 2015;11:491-7. https://doi.org/10.1200/ JOP.2015.004929.

23. Ramamoorthy A, Knepper TC, Merenda C, Mendoza M, McLeod HL, Bull J, et al. demographic composition of select oncologic new molecular entities approved by the FDA between 2008 and 2017. Clin Pharmacol Ther. 2018;104:940-8. https://doi. org/10.1002/cpt.1180. 
24. Elting LS, Cooksley C, Bekele BN, Frumovitz M, Avritscher EB, Sun C, et al. Generalizability of cancer clinical trial results: prognostic differences between participants and nonparticipants. Cancer. 2006;106:2452-8. https://doi.org/10.1002/cncr.21907.

25. Rothwell PM. Commentary: external validity of results of randomized trials: disentangling a complex concept. Int J Epidemiol. 2010;39:94-6. https://doi.org/10.1093/ije/dyp305.

26. Dekkers OM, von Elm E, Algra A, Romijn JA, Vandenbroucke JP. How to assess the external validity of therapeutic trials: a conceptual approach. Int J Epidemiol. 2010;39:89-94. https:// doi.org/10.1093/ije/dyp174.

27. Argyropulo-Palmer M, Jenkins A, Theti DS, Larkin J, Montgomery D. Sunitinib in metastatic renal cell carcinoma: a systematic review of UK real world data. Front Oncol. 2015;5:195. https:// doi.org/10.3389/fonc.2015.00195.

28. Liberati A, Altman DG, Tetzlaff J, Mulrow C, Gotzsche PC, Ioannidis JP, et al. The PRISMA statement for reporting systematic reviews and meta-analyses of studies that evaluate health care interventions: explanation and elaboration. J Clin Epidemiol. 2009;62:e1-34. https://doi.org/10.1016/j.jclinepi.2009.06.006.

29. Morrison A, Polisena J, Husereau D, Moulton K, Clark M, Fiander $\mathrm{M}$, et al. The effect of English-language restriction on systematic review-based meta-analyses: a systematic review of empirical studies. Int J Technol Assess Health Care. 2012;28:138-44. https ://doi.org/10.1017/S0266462312000086.

30. Armstrong AJ, Halabi S, Eisen T, Broderick S, Stadler WM, Jones $\mathrm{RJ}$, et al. Everolimus versus sunitinib for patients with metastatic non-clear cell renal cell carcinoma (ASPEN): a multicentre, openlabel, randomised phase 2 trial. Lancet Oncol. 2016;17:378-88. https://doi.org/10.1016/S1470-2045(15)00515-X.

31. Choueiri TK, Halabi S, Sanford BL, Hahn O, Michaelson MD, Walsh MK, et al. Cabozantinib versus sunitinib as initial targeted therapy for patients with metastatic renal cell carcinoma of poor or intermediate risk: the alliance A031203 CABOSUN trial. J Clin Oncol. 2017;35:591-7. https://doi.org/10.1200/ JCO.2016.70.7398.

32. Motzer RJ, Barrios CH, Kim TM, Falcon S, Cosgriff T, Harker WG, et al. Phase II randomized trial comparing sequential firstline everolimus and second-line sunitinib versus first-line sunitinib and second-line everolimus in patients with metastatic renal cell carcinoma. J Clin Oncol. 2014;32:2765-72. https://doi. org/10.1200/JCO.2013.54.6911.

33. Motzer RJ, Hutson TE, Cella D, Reeves J, Hawkins R, Guo J, et al. Pazopanib versus sunitinib in metastatic renal-cell carcinoma. N Engl J Med. 2013;369:722-31. https://doi.org/10.1056/NEJMo a1303989.

34. Motzer RJ, Hutson TE, Olsen MR, Hudes GR, Burke JM, Edenfield WJ, et al. Randomized phase II trial of sunitinib on an intermittent versus continuous dosing schedule as first-line therapy for advanced renal cell carcinoma. J Clin Oncol. 2012;30:1371-7. https://doi.org/10.1200/JCO.2011.36.4133.

35. Rini BI, Stenzl A, Zdrojowy R, Kogan M, Shkolnik M, Oudard S, et al. IMA901, a multipeptide cancer vaccine, plus sunitinib versus sunitinib alone, as first-line therapy for advanced or metastatic renal cell carcinoma (IMPRINT): a multicentre, open-label, randomised, controlled, phase 3 trial. Lancet Oncol. 2016;17:1599611. https://doi.org/10.1016/S1470-2045(16)30408-9.

36. Bamias A, Karadimou A, Lampaki S, Lainakis G, Malettou L, Timotheadou E, et al. Prognostic stratification of patients with advanced renal cell carcinoma treated with sunitinib: comparison with the Memorial Sloan-Kettering prognostic factors model. BMC Cancer. 2010;10:45. https://doi. org/10.1186/1471-2407-10-45.

37. Day D, Kanjanapan Y, Kwan E, Yip D, Lawrentschuk N, Andrews $\mathrm{M}$, et al. Patterns of care for metastatic renal cell carcinoma in Australia. BJU Int. 2015;116(Suppl 3):36-41. https://doi. org/10.1111/bju.13176.

38. De Giorgi U, Scarpi E, Sacco C, Aieta M, Lo Re G, Sava T, et al. Standard vs adapted sunitinib regimen in elderly patients with metastatic renal cell cancer: results from a large retrospective analysis. Clin Genitourin Cancer. 2014;12:182-9. https://doi. org/10.1016/j.clgc.2013.11.005.

39. De Groot S, Sleijfer S, Redekop WK, Oosterwijk E, Haanen JB, Kiemeney LA, et al. Variation in use of targeted therapies for metastatic renal cell carcinoma: results from a Dutch population-based registry. BMC Cancer. 2016;16:364. https://doi. org/10.1186/s12885-016-2395-x.

40. Heng DY, Chi KN, Murray N, Jin T, Garcia JA, Bukowski RM, et al. A population-based study evaluating the impact of sunitinib on overall survival in the treatment of patients with metastatic renal cell cancer. Cancer. 2009;115:776-83. https://doi. org/10.1002/cncr.24051.

41. Lalani AA, Li H, Heng DYC, Wood L, Kalirai A, Bjarnason GA, et al. First-line sunitinib or pazopanib in metastatic renal cell carcinoma: the Canadian experience. Can Urol Assoc J. 2017;11:112-7. https://doi.org/10.5489/cuaj.4398.

42. Maroun R, Fleury L, Nachbaur G, Maunoury F, Vanhille JL, Durand-Zaleski I. Real-world costs and outcomes in metastatic renal cell carcinoma patients treated with targeted therapies: a cohort study from the French health insurance database. Curr Med Res Opin. 2017;33:1755-62. https://doi.org/10.1080/03007 995.2017.1360850.

43. Miyake H, Miyazaki A, Harada K, Fujisawa M. Assessment of efficacy, safety and quality of life of 110 patients treated with sunitinib as first-line therapy for metastatic renal cell carcinoma: experience in real-world clinical practice in Japan. Med Oncol. 2014;31:978. https://doi.org/10.1007/s12032-014-0978-4.

44. Noize P, Grelaud A, Bay JO, Chevreau C, Gross-Goupil M, Culine S, et al. Real-life patterns of use, safety and effectiveness of sunitinib in first-line therapy of metastatic renal cell carcinoma: the SANTORIN cohort study. Pharmacoepidemiol Drug Saf. 2017;26:1561-9. https://doi.org/10.1002/pds.4228.

45. Pan X, Huang H, Huang Y, Liu B, Cui X, Gan S, et al. Sunitinib dosing schedule $2 / 1$ improves tolerability, efficacy, and healthrelated quality of life in Chinese patients with metastatic renal cell carcinoma. Urol Oncol. 2015;33(268):e9-15. https://doi. org/10.1016/j.urolonc.2015.03.008.

46. Poprach A, Lakomy R, Bortlicek Z, Melichar B, Pavlik T, Slaby $\mathrm{O}$, et al. Efficacy of sunitinib in elderly patients with metastatic renal cell carcinoma: data from real-world clinical practice. Drugs Aging. 2016;33:655-63. https://doi.org/10.1007/s4026 6-016-0390-1.

47. Schnadig ID, Hutson TE, Chung H, Dhanda R, Halm M, Forsyth $\mathrm{M}$, et al. Dosing patterns, toxicity, and outcomes in patients treated with first-line sunitinib for advanced renal cell carcinoma in community-based practices. Clin Genitourin Cancer. 2014;12:413-21. https://doi.org/10.1016/j.clgc.2014.06.015.

48. Sheng X, Chi Z, Cui C, Si L, Li S, Tang B, et al. Efficacy and safety of sorafenib versus sunitinib as first-line treatment in patients with metastatic renal cell carcinoma: largest single-center retrospective analysis. Oncotarget. 2016;7:27044-54. https://doi. org/10.18632/oncotarget.7395.

49. Tan HS, Li H, Hong YW, Toh CK, Wong A, Lopes G, et al. Efficacy and safety of an attenuated-dose sunitinib regimen in metastatic renal cell carcinoma: results from a prospective registry in singapore. Clin Genitourin Cancer. 2015;13:e285-95. https://doi. org/10.1016/j.clgc.2014.11.004.

50. Zhang HL, Sheng XN, Li XS, Wang HK, Chi ZH, He ZS, et al. Sorafenib versus sunitinib as first-line treatment agents in Chinese patients with metastatic renal cell carcinoma: the largest multicenter retrospective analysis of survival and prognostic 
factors. BMC Cancer. 2017;17:16. https://doi.org/10.1186/s1288 5-016-3016-4.

51. Ruiz-Morales JM, Swierkowski M, Wells JC, Fraccon AP, Pasini F, Donskov F, et al. First-line sunitinib versus pazopanib in metastatic renal cell carcinoma: results from the International Metastatic Renal Cell Carcinoma Database Consortium. Eur J Cancer. 2016;65:102-8. https://doi.org/10.1016/j.ejca.2016.06.016.

52. Pal SK, Ghate SR, Li N, Swallow E, Peeples M, Zichlin ML, et al. Real-world survival outcomes and prognostic factors among patients receiving first targeted therapy for advanced renal cell carcinoma: a SEER-medicare database analysis. Clin Genitourin Cancer. 2017;15:e573-82. https://doi.org/10.1016/j. clgc.2016.12.005

53. Pearce W, Raman S, Turner A. Randomised trials in context: practical problems and social aspects of evidence-based medicine and policy. Trials. 2015;16:394. https://doi.org/10.1186/s1306 3-015-0917-5.

54. Amin A, Plimack ER, Ernstoff MS, Lewis LD, Bauer TM, McDermott DF, et al. Safety and efficacy of nivolumab in combination with sunitinib or pazopanib in advanced or metastatic renal cell carcinoma: the CheckMate 016 study. J Immunother Cancer. 2018;6:109. https://doi.org/10.1186/s40425-018-0420-0.

55. Fiedler W, Serve H, Dohner H, Schwittay M, Ottmann OG, O'Farrell AM, et al. A phase 1 study of SU11248 in the treatment of patients with refractory or resistant acute myeloid leukemia (AML) or not amenable to conventional therapy for the disease. Blood. 2005;105:986-93. https://doi.org/10.1182/blood -2004-05-1846.

56. Faivre S, Delbaldo C, Vera K, Robert C, Lozahic S, Lassau N, et al. Safety, pharmacokinetic, and antitumor activity of SU11248, a novel oral multitarget tyrosine kinase inhibitor, in patients with cancer. J Clin Oncol. 2006;24:25-35. https://doi.org/10.1200/ JCO.2005.02.2194.

57. Boegemann M, Hubbe M, Thomaidou D, Blackburn S, BentEnnakhil N, Wood R, et al. Sunitinib treatment modification in first-line metastatic renal cell carcinoma: analysis of the STARTOR registry. Anticancer Res. 2018;38:6413-22. https://doi. org/10.21873/anticanres.13002.

58. Lee JL, Kim MK, Park I, Ahn JH, Lee DH, Ryoo HM, et al. RandomizEd phase II trial of Sunitinib four weeks on and two weeks off versus Two weeks on and One week off in metastatic clear-cell type REnal cell carcinoma: RESTORE trial. Ann Oncol. 2015;26:2300-5. https://doi.org/10.1093/annonc/mdv357.
59. Fujita T, Hirayama T, Ishii D, Matsumoto K, Yoshida K, Iwamura M. Efficacy and safety of sunitinib in elderly patients with advanced renal cell carcinoma. Mol Clin Oncol. 2018;9:394-8. https://doi.org/10.3892/mco.2018.1684.

60. Miyake H, Aki R, Matsushita Y, Tamura K, Motoyama D, Ito T, et al. Significance of age in Japanese patients receiving sunitinib as first-line systemic therapy for metastatic renal cell carcinoma: comparative assessment of efficacy and safety between patients aged $<75$ and $>/=75$ years. Anticancer Res. 2018;38:3593-9. https://doi.org/10.21873/anticanres.12633.

61. Hutson TE, Bukowski RM, Rini BI, Gore ME, Larkin JM, Figlin RA, et al. Efficacy and safety of sunitinib in elderly patients with metastatic renal cell carcinoma. Br J Cancer. 2014;110:1125-32. https://doi.org/10.1038/bjc.2013.832.

62. Guo J, Jin J, Oya M, Uemura H, Takahashi S, Tatsugami K, et al. Safety of pazopanib and sunitinib in treatment-naive patients with metastatic renal cell carcinoma: Asian versus non-Asian subgroup analysis of the COMPARZ trial. J Hematol Oncol. 2018;11:69. https://doi.org/10.1186/s13045-018-0617-1.

63. Gore ME, Szczylik C, Porta C, Bracarda S, Bjarnason GA, Oudard $\mathrm{S}$, et al. Final results from the large sunitinib global expandedaccess trial in metastatic renal cell carcinoma. Br J Cancer. 2015;113:12-9. https://doi.org/10.1038/bjc.2015.196.

64. Heng DY, Choueiri TK, Rini BI, Lee J, Yuasa T, Pal SK, et al. Outcomes of patients with metastatic renal cell carcinoma that do not meet eligibility criteria for clinical trials. Ann Oncol. 2014;25:149-54. https://doi.org/10.1093/annonc/mdt492.

65. Marschner N, Staehler M, Muller L, Nusch A, Harde J, Koska M, et al. Survival of patients with advanced or metastatic renal cell carcinoma in routine practice differs from that in clinical trials-analyses from the german clinical RCC registry. Clin Genitourin Cancer. 2017;15:e209-15. https://doi.org/10.1016/j. clgc.2016.08.022.

66. Nieder C, Syed MA, Dalhaug A, Pawinski A, Norum J. Eligibility for phase 3 clinical trials of systemic therapy in real-world patients with metastatic renal cell cancer managed in a rural region. Med Oncol. 2017;34:149. https://doi.org/10.1007/s12032-017-1002-6.

67. Fernandez-Pello S, Hofmann F, Tahbaz R, Marconi L, Lam TB, Albiges L, et al. A systematic review and meta-analysis comparing the effectiveness and adverse effects of different systemic treatments for non-clear cell renal cell carcinoma. Eur Urol. 2017;71:426-36. https://doi.org/10.1016/j.eururo.2016.11.020. 